\title{
AVALIAÇÃO DA TEMPERATURA DE BRILHO NOS CANAIS SENSÍVEIS À SUPERFÍCIE TERRESTRE NO SISTEMA G3DVAR DO CPTEC/INPE: SÉRIE NOAA
}

\author{
BRUNNA ROMERO PENNA, BRUNA BARBOSA SILVEIRA, LUIS GUSTAVO GONÇALVES DE \\ GONÇALVES, SOLANGE SILVA DE SOUZA
}

\author{
Instituto Nacional de Pesquisas Espaciais, Centro de Previsão de Tempo e Estudos Climáticos, Cachoeira \\ Paulista, SP, Brasil
}
brunnaromeropenna@gmail.com, bruna.silveira@cptec.inpe.br, gustavo.goncalves@cptec.inpe.br, solange.souza@cptec.inpe.br

Recebido Fevereiro de 2014 - Aceito Janeiro de 2015

\begin{abstract}
RESUMO
As medidas do AMSU-A para os canais que são sensíveis à superfície terrestre sobre os continentes não tem sido amplamente utilizadas para ajustar a previsão numérica de tempo de curto prazo (PNTs), devido à complexidade das características da superfície terrestre. Nesse sentido, o presente artigo utiliza o Sistema de Assimilação de Dados (G3DVAR) do Centro de Previsão de Tempo e Estudos Climáticos do Instituto Nacional de Pesquisas Espaciais (CPTEC/INPE), que inclui tais medidas de radiâncias feitas pelo sensor que está a bordo dos satélites da série NOAA. A versão operacional do sistema G3DVAR contempla apenas o satélite NOAA-15. Adicionalmente, foram realizados experimentos numéricos que incluíram os satélites NOAA-18 e NOAA-19. É feita uma avaliação sobre a variável temperatura de brilho simulada para os canais sensíveis à superfície terrestre (i), através de uma comparação com observações, e (ii) através da avaliação da equação de transferência radiativa, para os três satélites. Os resultados indicaram que o modelo de transferência radiativa em média superestima a temperatura de brilho nos canais sensíveis à superfície terrestre para os três satélites na região da América do Sul para os meses de verão. Além disso, as observações dos satélites incorporadas no sistema tiveram um aceite superior ao do satélite NOAA-15, de maneira que os satélites NOAA-18 e NOAA-19 podem ser incorporados no modo operacional do Sistema G3DVAR.
\end{abstract}

Palavras-chave: Assimilação de dados, radiância, observação, simulação, AMSU-A, NOAA.

\begin{abstract}
EVALUATION OF THE WINDOWS CHANNEL BRIGHTNESS TEMPERATURE IN G3DVAR SYSTEM OF CPTEC/INPE: SERIE NOAA

Channels sensitive to the terrestrial surface have not been widely used in Numerical Weather Prediction (NWP) due to the inherent complexities of the land surface. Thus, the present paper tested the tridimensional variational data assimilation framework implemented in the Global Model (G3DVAR) at the Center for Weather Forecast and Climate Studies (CPTEC) from the National Institute for Space Research (INPE) with near surface radiances from the NOAA-15 satellite. Since the operational version of the G3DVAR uses only NOAA-15, other experiments including the NOAA-18 and NOAA-19 satellites were performed. An assessment of the simulated brightness temperature for the near surface channels was made (i) through a comparison against observations and (ii) through a validation of the radiative transfer equation for the three satellites. The results indicate that the radiative transfer model overestimate, on average, the brightness temperature from the channels sensitive to the earth surface for all the considered satellites over South America during the austral summer. Furthermore, the number of observations accepted by the assimilation system increased substantially when NOAA-18 and NOAA-19 satellites were included, suggesting that incorporating these two new satellites should bring a positive impact to the G3DVAR operational system at CPTEC/INPE.
\end{abstract}

Keywords: data assimilation, radiance, observation, simulation, AMSU-A, NOAA. 


\section{INTRODUÇÃO}

Durante as últimas décadas houve uma evolução significativa na quantidade e qualidade de observações meteorológicas, sejam estas convencionais (estações de superfície, ar superior, etc.) ou não convencionais (provenientes de sensoriamento remoto da atmosfera), assim como um grande avanço na qualidade dos modelos utilizados em Previsão Numérica de Tempo (PNT). Este último associado à melhoria nas parametrizações de sub-grade e ao aumento de resolução. Entretanto, somente durante os últimos anos é que a combinação destas duas informações tem apresentado uma evolução mais significativa para a meteorologia, com a utilização de técnicas avançadas de assimilação de dados. A assimilação de dados através de um processo físico-estatístico combina as informações de modelos numéricos (uma estimativa inicial) e as observações meteorológicas, gerando a melhor representação possível do estado da atmosfera em um dado instante de tempo (Herdies et al., 2008).

As previsões meteorológicas são realizadas executando (integrando no tempo) modelos computacionais que simulam a atmosfera dado as condições iniciais da mesma, ou seja, o tempo de integração de um modelo atmosférico é um problema de valor inicial e a capacidade de fazer uma previsão hábil exige, tanto um modelo, com uma representação realística da atmosfera, como condições iniciais que sejam conhecidas de forma satisfatória. Nos últimos 50 anos houve uma melhoria na habilidade de PNT devido a fatores como o aumento do poder computacional, a melhoria nas representações dos processos físicos de pequena escala e o uso de métodos mais precisos de assimilação de dados, o que resulta em melhores condições iniciais para os modelos e aumento na disponibilidade de dados, especialmente dados de satélites e aviões ao longo dos oceanos e do hemisfério sul (Kalnay, 2003).

O Centro de Previsão de Tempo e Estudos Climáticos do Instituto Nacional de Pesquisas Espaciais (CPTEC/INPE) opera desde 2013 o Sistema de Assimilação de Dados nomeado G3DVAR, cuja composição é o Modelo de Circulação Geral da Atmosfera (MCGA) e o método estatístico variacional tridimensional (3DVAR), o Gridpoint Statistical Interpolation (GSI). O objetivo do G3DVAR é produzir as condições iniciais para as previsões de curto e médio prazos do modelo do CPTEC (Gonçalves et al., 2015). Os tipos de observações assimiladas pelo sistema podem ser tanto convencionais como não convencionais. As observações não convencionais são os dados de satélites, pois elas não medem as variáveis de estado do modelo, mas sim radiância, a qual constitui a abordagem do presente documento.

Um dos primeiros trabalhos realizados no CPTEC/INPE foi de Aravéquia (2008), que descreve a implantação de um modelo de transferência radiativa para permitir a assimilação de dados de radiância no sistema de análise Local Ensemble Transform Kalman Filter (LETKF). Mostrou também qual é o impacto da assimilação destas observações de alguns canais do sensor Atmospheric Infrared Sounder (AIRS) na análise inicial do modelo. Por conseguinte, Medeiros (2010) avaliou o desempenho do LETKF na assimilação de dados convencionais e de radiância do sensor AMSU-A. Ambos apresentaram impactos positivos nas previsões e análises com a assimilação de radiâncias, mostrando a relevância deste tipo de observação em um sistema de assimilação.

A assimilação de radiâncias de sensores de micro-ondas, tal como o sensor utilizado por Medeiros (2010), para os canais sensíveis às condições da superfície terrestre, depende da diferença entre a radiância observada pelo satélite e a simulada pelo Modelo de Transferência Radiativa (MTR).No sistema de assimilação G3DVAR, o modelo MTR é o Community Radiative Transfer Model (CRTM), e este simula a radiância com base nas variáveis fornecidas pelo modelo MCGA. Assim, o sistema de assimilação de dados aplica critérios sobre a radiância observada, conforme um controle de qualidade, em que, por exemplo, estabelecido um valor máximo entre a diferença das radiâncias observada e simulada tem-se a inclusão ou exclusão do dado observado.

A radiância representa a energia resultante no sistematerra atmosfera, quando vista através do satélite, e tem a contribuição da temperatura e emissividade da superfície, da transmitância e profundidade óptica da atmosfera, dentre outros. A dificuldade em simular a emissividade e a temperatura da superfície terrestre foi verificada por Zheng et al. (2012). Ademais, recentes trabalhos tem apontado a temperatura da superfície terrestre como um fator chave na assimilação de dados de satélites (Zheng et al., 2009 ; He et al., 2011 ; Karbou et al., 2007).

Especificamente, a temperatura da superfície terrestre do G3DVAR provém do modelo biofísico de superfície, o Simplified Simple Biosphere Model (SSiB) (Xue et al., 1991), cujo acoplamento online com o modelo MCGA trata das interações superfície-atmosfera. O propósito deste modelo é simular realisticamente o controle dos processos biofísicos e fornecer os fluxos de radiação, de momentum, de calor sensível e de calor latente para o modelo atmosférico. O modelo SSiB possui um esquema de cálculo para temperatura da superfície terrestre que depende da radiação de onda longa emitida pela superfície, onde a radiação de onda longa emitida depende da formulação das temperaturas do dossel e do solo resolvidos.

Atualmente no sistema G3DVAR estão sendo assimilados dados de radiância dos canais sensíveis à superfície somente do satélite National Oceanic and Atmospheric Adimistration Unit - 15 (NOAA-15). Entretanto, como o volume de dados 
assimilados sobre o continente é bem inferior àqueles sobre o oceano, devido primariamente às características complexas da superfície terrestre, conforme notado por Kazumori (2012), há necessidade de inclusão de dados. Assim, existe um esforço para assimilar todos os dados dos satélites NOAA que tem a bordo o sensor AMSU-A, tal como, os satélites NOAA-18 e NOAA-19.

Corroborando com a verificação do Sistema de Assimilação de Dados G3DVAR do CPTEC/INPE, o presente documento avaliou a radiância simulada nos canais sensíveis à superfície terrestre. A radiância pode ser expressa como temperatura de brilho e é oriunda do modelo CRTM e das observações proveniente do sensor de micro-ondas AMSU-A a bordo dos satélites NOAA-15, NOAA-18 e NOAA-19. No modelo é aplicada a equação de energia de Planck (Liou, 1980), considerando componentes dos balanços de energia à superfície terrestre e na atmosfera.

A estrutura do trabalho foi montada como segue. $\mathrm{Na}$ seção 2 são apresentadas as especificações do Sistema G3DVAR, como os dados de entrada, o modelo de PNT, o modelo estatístico que acopla ambas as informações, o modelo de radiância CRTM, bem como, o tratamento sobre o parâmetro de interesse. $\mathrm{Na}$ seção 3, são apresentados os resultados e discussões; na seção 4 , as conclusões e recomendações.

\section{MATERIAIS E MÉTODOS}

\subsection{Dados}

O Sistema de Assimilação de Dados G3DVAR, basicamente utiliza dados convencionais e não convencionais do pacote PrepBUFR (Prepared Binary Universal Form for the Representation of meteorological data) do National Centers for Environmental Prediction (NCEP), conforme documentado (Souza et al., 2010), dados de GPS (Global Navigation System) e de radiância são do pacote BUFR (Binary Universal Form for the Representation of meteorological data). Esses dados são tratados pelo sistema no incremento da análise (anl), como correção a estimativa inicial (ges, do inglês first guess).

Os dados de radiância provêm de diversos sensores a bordo de satélites geoestacionários e polares, e são tratados como temperatura de brilho. No presente trabalho são inspecionados os dados coletados pelos sensores AMSU-A a bordo dos satélites NOAA-15, NOAA-18 e NOAA-19. Esses satélites de órbita quase polar e heliossíncrona fornecem informações duas vezes por dia, durante as passagens ascendente e descendente, estão numa altitude em torno de $840 \mathrm{~km}$, abrangem uma faixa de largura de $2345 \mathrm{~km}$ em cada linha de varredura e cuja órbita sobre o globo tem um período de 102 minutos.

Este instrumento observa a Terra com um ângulo de digitalização que varia de $-48^{\circ} \mathrm{a}+48^{\circ}$ (Karbou et al., 2007), com um total de 30 campos de visualização por linha de varredura . A leitura ocorre nas polarizações horizontal e vertical. O sensor de micro-ondas possui medidas em 15 frequências abrangendo a faixa espectral de 23,8 a $89,0 \mathrm{GHz}$. Os canais sensíveis à superfície e respectivas características, como frequência, número do canal correspondente e precisão são apresentados na Tabela 1. No Nadir, o sensor fornece o dado numa resolução espacial em torno de $48 \mathrm{~km}$ e numa polarização vertical para os canais de interesse.

\subsection{O Sistema de G3DVAR}

O ciclo de assimilação foi executado a cada 6 horas, com início às $0000 \mathrm{Z}$ de 01 de novembro de 2012 e término em 30 de abril de 2013, em que foram geradas 724 análises diárias nos horários sinóticos $(0000,0600,1200$ e 1800 Z). A estimativa inicial foi oriunda da análise do NCEP, das $1800 \mathrm{Z}$ de 31 de outubro de 2012. Os dois primeiros meses foram excluídos das análises em virtude do período de equilíbrio dinâmico (do inglês spin-up), de modo que são consideradas as saídas a partir de $0000 \mathrm{Z}$ de 01 de janeiro 2013. O experimento foi realizado no Tupã, sistema de supercomputação adquirido pelo INPE, que é composto por um supercomputador Cray XE6, e está instalado nas dependências do CPTEC/INPE. No total geral, o sistema G3DVAR demandou um tempo de 483 horas e um espaço em disco de $770 \mathrm{~Gb}$

\section{i) O Modelo MCGA}

As informações de estimativa inicial no sistema G3DVAR provêm do modelo MCGA do CPTEC/INPE (Kubota, 2012 e Bonatti, 1996) na resolução T0299L64, onde T0299 refere-se ao truncamento triangular na onda zonal 299 e L64 refere-se ao número de camadas verticais de 64 níveis. No equador a resolução espacial é de aproximadamente $44 \mathrm{~km}$.

Sobre o continente, o modelo atmosférico está acoplado ao modelo biofísico de superfície SSiB (Xue et al., 1991). A junção de ambos permite as trocas de quantidades de radiação, de calor e de movimento entre a superfície e a atmosfera. A vegetação (biomas) é tratada explicitamente e a temperatura da superfície terrestre resulta das frações de áreas vegetada e

Tabela 1: Características dos canais do AMSU-A sensíveis à superfície terrestre.

\begin{tabular}{ccc}
\hline Número do Canal & Frequência (GHz) & Calibração/Precisão (k) \\
\hline 1 & 23,8 & 2,0 \\
\hline 2 & 31,4 & 2,0 \\
\hline 3 & 50,3 & 1,5 \\
\hline 15 & 89,0 & 2,0
\end{tabular}

Fonte: Adaptada de http://mirs.nesdis.noaa.gov/ASua.php 
não vegetada em cada célula, sendo uma função da radiação de onda longa. A radiação de onda longa emitida pela superfície continental tem contribuição de dois termos: i) temperatura do dossel; e ii) temperatura do solo, nos dois primeiros centímetros. Em solos nus, a radiação de onda longa emitida perde o termo devido ao dossel.

\section{ii) O Esquema GSI}

O esquema GSI foi desenvolvido no NCEP, como parte de um esforço para criar um esquema de análise mais unificado, robusto e eficiente. Como aspecto fundamental, a formulação da análise é feita no espaço de grade do modelo, o que permite uma maior flexibilidade na aplicação das covariâncias do erro da estimativa inicial e um sistema de análise único pode ser facilmente usado em uma gama de aplicações, incluindo os sistemas de modelagem nos domínios global e regional (Kleist et al., 2009). O sistema variacional 3DVAR é resolvido pela função de custo $\mathrm{J}$ (Hu et al., 2013):

$$
\begin{aligned}
& J=\left(x_{a}-x_{b}\right)^{T} B^{-1}\left(x_{a}-x_{b}\right)+\left(H x_{a}-o_{o}\right)^{T} . \\
& R^{-1}\left(H x_{a}-o_{o}\right)+J_{c},
\end{aligned}
$$

onde, representa o vetor de estado do campo de análise, o campo da estimativa inicial, é a matriz de covariância do erro da estimativa inicial, é o operador de observação não linear, são as observações, é a matriz de covariância do erro das observações, é o termo relacionado com erros no modelo de PNT. O termo representa a variável do modelo no espaço das observações. Assim, é o operador cujo papel é interpolar espacialmente as variáveis para o caso dos dados convencionais, e é o MTR para os dados não convencionais.

\section{iii) O Modelo CRTM}

O modelo CRTM é disponibilizado pelo Joint Center for Satellite Data Assimilation (JCSDA) da NOAA, representa o operador de observação de radiância e é expresso como $(\mathrm{Hu}$ et al., 2013):

$$
y=K(x, Z),
$$

em que, é a radiância simulada, é o perfil de temperatura, umidade e ozônio, é o modelo CRTM e são os parâmetros não conhecidos como emissividade da superfície, perfil de $\mathrm{CO}_{2}$, metano, entre outros. O perfil (desde a superfície) provém da estimativa inicial e decorre do modelo de PNT. Quando as incógnitas em são muito grandes, seja pela formulação de ou pelos parâmetros desconhecidos, os dados de radiância não passam pelo controle de qualidade. Na Figura 1 observa-se um diagrama esquemático simplificado de todo o processo de assimilação de dados. Ressalta-se que na etapa de thinning dos dados são selecionados dados espaçados a cada $145 \mathrm{~km}$ para o sensor AMSU-A.

Resumidamente, os componentes dos processos de transferência radiativa considerado pelo modelo CRTM são divididos em quatro categorias principais (Han. et al., 2005): i) absorção de radiação pelos constituintes gasosos da atmosfera, ii) absorção e espalhamento de radiação por nuvens e aerossóis, iii) superfície de emissão de radiação e de interação de superfície com subsidência de radiação atmosférica, iv) solução da equação de transferência radiativa.

\section{iv) Radiâncias}

Para uma atmosfera plano-paralela e para um dado caminho óptico, um ângulo zenital de observação () e uma frequência de onda () do sensor AMSU-A tem a seguinte relação (Karbou et al., 2006):

$$
\begin{aligned}
& T_{b}(\nu, \theta)=T_{s} \varepsilon(\nu, \theta) \Gamma+\{1-\varepsilon(\nu, \theta)\} \Gamma T_{a}^{\downarrow}(\nu, \theta)+T_{a}^{\uparrow}(v, \theta) \\
& \Gamma=\exp \left\{\frac{-\tau(0, h)}{\cos \theta}\right\},
\end{aligned}
$$

em que, os parâmetros $T_{b}, \varepsilon, T_{a}^{\downarrow}$ e $T_{a}^{\uparrow}$ são, respectivamente, a temperatura de brilho, a emissividade da superfície terrestre, a temperatura incidente na atmosfera e a temperatura emergente na atmosfera. A relação foi estabelecida para a obtenção de uma

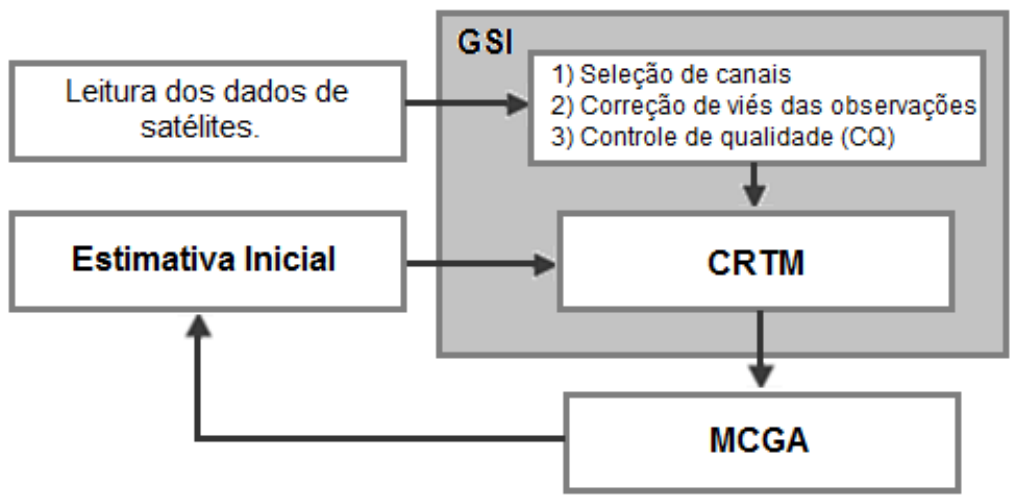

Figura 1: Diagrama esquemático simplificado de todo o processo de assimilação de dados de radiância no sistema G3DVAR. Fonte: Adaptado de Silveira (2012). 
em unidades de Kelvin, ou seja, as temperaturas envolvidas na Equação 3 são expressas em Kelvin. A variável é a temperatura da superfície terrestre; , transmissividade atmosférica líquida e incorpora a opacidade atmosférica, desde a superfície até o topo da atmosfera $(h)$. Nota-se que participa das energias provenientes da superfície e da radiação incidente sobre a atmosfera. A emissividade da superfície terrestre foi calculada conforme o modelo de Weng et al. (2001), nas polarizações vertical e horizontal, e tem como parâmetros de entrada o teor de umidade do solo, a fração de área vegetada, a temperatura do solo, a temperatura da superfície terrestre e a profundidade da neve. A parametrização da emissividade, como já mencionado acima, faz parte do modelo CRTM, estando na categoria iii.

\subsection{Controle de Qualidade de Radiância Observada}

O Controle de Qualidade (CQ) é inserido no sistema GSI porque os dados de radiância, observada no arquivo em formato BUFR, não incluem a informação da qualidade da observação (como o que acontece com os dados no arquivo prepBUFR, o qual contém as marcas do controle de qualidade das observações). No sistema de assimilação, o CQ inicia após a leitura da radiância observada, onde o processo de afinamento sobre os dados, chamado thinning, é aplicado para evitar a redundância de dados. Além disso, vários procedimentos são seguidos no $\mathrm{CQ}$, os quais são descritos abaixo para deter os dados de satélites problemáticos, que provêm principalmente de (i) problemas do sensor, (ii) erros de simulação de nuvens e de precipitação, (iii) erros na simulação de emissividade da superfície e (iv) erros de processamento (p.e., atribuição errada da altura e rastreamento incorreto). No conjunto de correções estão definidas 7 categorias sobre os pixels, para cada instrumento. A primeira correção, CQ1, rejeita os dados dos canais 1-6 e 15, quando o parâmetro empírico que leva em consideração a observação e simulação do canal 4 e o conteúdo de agua líquida de nuvens for superior a 0,5. A segunda correção, CQ2, leva em conta a acurácia da estimativa da emissividade e da temperatura da superfície oceânica para rejeitar dados dos mesmos canais do CQ1. O CQ3 rejeita dados dos canais 1-7 e 15, quando o parâmetro empírico que leva em consideração a observação e simulação do canal 6 e o índice de espalhamento for superior a 1. O CQ4 e o CQ5 levam em consideração a altura do terreno referente ao ponto da observação. Desta maneira quando a altura do terreno for maior que 2000 m, o erro da observação do canal 7 é modificado, isto é, o erro é ampliado. O mesmo ocorre no CQ5 se a altura do terreno for superior a $4000 \mathrm{~m} \mathrm{o}$ erro relacionado à observação do canal 8 é ampliado. No CQ6 se a água líquida de nuvens for superior a 1,0, a observação é contabilizada com esta categoria, o CQ6 faz parte do CQ1, pois a medida de água líquida de nuvem faz parte do cálculo do parâmetro empírico do CQ1. Já no CQ7, se o índice de espalhamento for superior a 1,0, a observação é contabilizada nesta categoria. Como o CQ6, o CQ7 faz parte do CQ3, pois o índice de espalhamento é utilizado no cálculo do parâmetro empírico do CQ3. A Tabela 2 mostra resumidamente as categorias aplicadas sobre as observações do sensor AMSU-A.

Vale ressaltar que as radiâncias assimiladas pelo sistema de assimilação consideram apenas pixels que não estão contaminados por nebulosidade (Hu et al., 2013), isto implica na ausência de dados em regiões com nebulosidade.

Antes de passarem pelo CQ, os dados de radiância passam por uma correção de viés que repara os desvios sistemáticos entre as radiâncias observadas e simuladas, e as fontes de erros podem ser provenientes de: i) instrumentos de satélite (como erros de calibração); ii) modelo CRTM; iii) estimativa inicial, devido a erros sistemáticos no modelo de PNT. Com relação aos erros instrumentais do satélite, aplica-se a correções de viés sobre a varredura e sobre a massa de ar.

A partir da correção de viés e da estimativa inicial, um marcador da observação de radiância é aplicado. Em seguida,

Tabela 2: Quadro esquemático do controle de qualidade do sensor AMSU-A.

\begin{tabular}{|l|l|l|}
\hline Categoria & Etapas do Controle de Qualidade & Ação sobre a radiância \\
\hline CQ1 & Perfil com nebulosidade (parâmetro empírico >0,5) & É rejeitada nos canais 1-6 e 15 \\
\hline CQ2 & $\begin{array}{l}\text { Falta de acurácia nas estimativas da emissividade/ } \\
\text { temperaturas da superfície sobre o oceano. }\end{array}$ & É rejeitada nos canais 1-6 e 15 \\
\hline CQ3 & $\begin{array}{l}\text { Perfil afetado por nuvem (índice de espalhamento no } \\
\text { canal 6 }>1,0)\end{array}$ & É rejeitada nos canais 1-7 e 15 \\
\hline CQ4 & $\begin{array}{l}\text { Erro de observação acentuado sobre terreno elevado } \\
\text { (altura }>\text { 2000m) }\end{array}$ & $\begin{array}{l}\text { É acentuado o erro da } \\
\text { observação no canal 7 }\end{array}$ \\
\hline CQ5 & $\begin{array}{l}\text { Erro de observação acentuado sobre terreno elevado } \\
\text { (altura }>4000 m)\end{array}$ & $\begin{array}{l}\text { É acentuado o erro da } \\
\text { observação no canal 8 }\end{array}$ \\
\hline CQ6 & Total de água líquida na nuvem $>1,0$ & Parte do CQ1 \\
\hline CQ7 & Índice de espalhamento $>1,0$ & Parte do CQ3 \\
\hline
\end{tabular}

Fonte: Adaptado de $\mathrm{Hu}$ et al., 2013. 
fazem-se verificações quantitativas das diferenças entre os valores de radiâncias observada e a simulada. Nesta etapa, são consideradas as radiâncias simuladas sobre regiões que se estendem além do ponto de observação, num raio de influência, para verificar a robustez do dado observado pontualmente. Desse modo, o modelo de PNT é decisivo na seleção do dado, uma vez que a estimativa inicial é considerada como a "verdade" e a diferença entre as temperaturas de brilho simulada e observada não pode ser excessiva. Após essas avalições quantitativas o dado de temperatura de brilho observado é então removido pelo sistema G3DVAR.

\subsection{Avaliações das radiâncias}

A temperatura de brilho é investigada de duas formas: subjetiva e objetiva. Na forma subjetiva foram avaliadas as temperaturas de brilho simulada (saída do modelo CRTM) e observada (proveniente do arquivo BUFR), antes e após a correção de viés do satélite, e as que passaram pelo CQ, como sejam, as que foram assimiladas pelo Sistema G3DVAR. Na forma objetiva, a abordagem é feita sobre os termos da Equação 3 e o objetivo é identificar a contribuição do primeiro termo -da temperatura e da emissividade da superfície terrestre, considerando a transmissividade igual a 1 . A análise abrange a região continental da América do Sul (AS, coord: $57^{\circ} \mathrm{S}$ a $15^{\circ} \mathrm{N}$ e $82^{\circ} \mathrm{W}$ a $32^{\circ} \mathrm{W}$ ) e inclui os horários sinóticos durante o período selecionado. Valores médios foram calculados para os três satélites.

\section{RESULTADOS E DISCUSSÕES}

\subsection{Temperaturas de Brilho Observada e Simulada}

A distribuição temporal da temperatura de brilho média sobre a região AS é discutida para os três satélites.

A Figura 2 mostra informações para o satélite NOAA-15 no canal 3. Sobre a região de estudo, o número de observações assimiladas oscilou em torno de 105 (Figura 2a). Nos demais canais, o número foi menor.: no canal 1, 80; no canal 2, 60 e no canal 15, 95 (figuras não mostradas). As oscilações na quantidade de dados assimilados provêm do CQ e da área de varredura do satélite. A varredura é uma função da trajetória do satélite e é dependente se a passagem é ascendente ou descendente. $\mathrm{O}$ satélite NOAA 15 passou sobre a AS nos 4 horários sinóticos e na passagem às $1200 \mathrm{Z}$ as informações se limitaram na porção noroeste do continente. Assim poucos dados estiveram disponíveis e nenhum dado foi assimilados em certas ocasiões. A oscilação do número de observações apresentou uma periodicidade 5-6 dias.

A Figura $2 b$ mostra a temperatura de brilho observada menos a temperatura de brilho da estimativa inicial. Em (a)

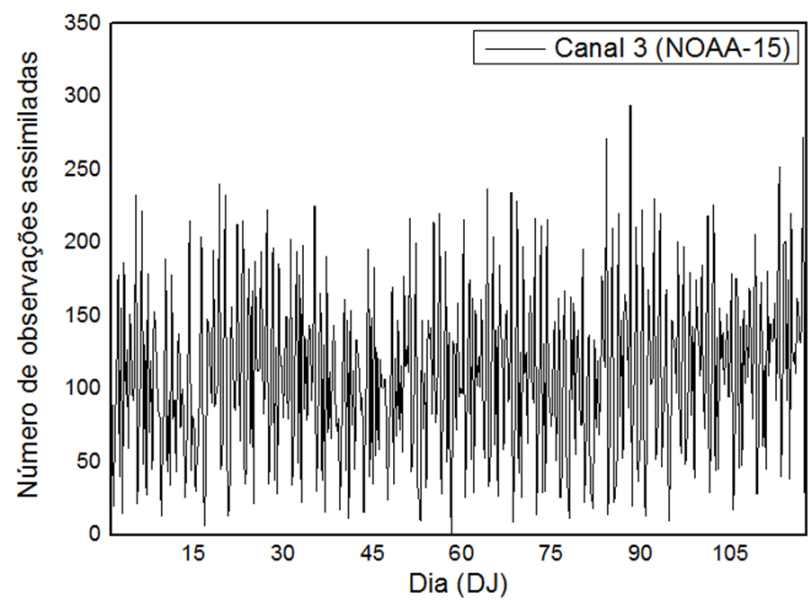

(b)

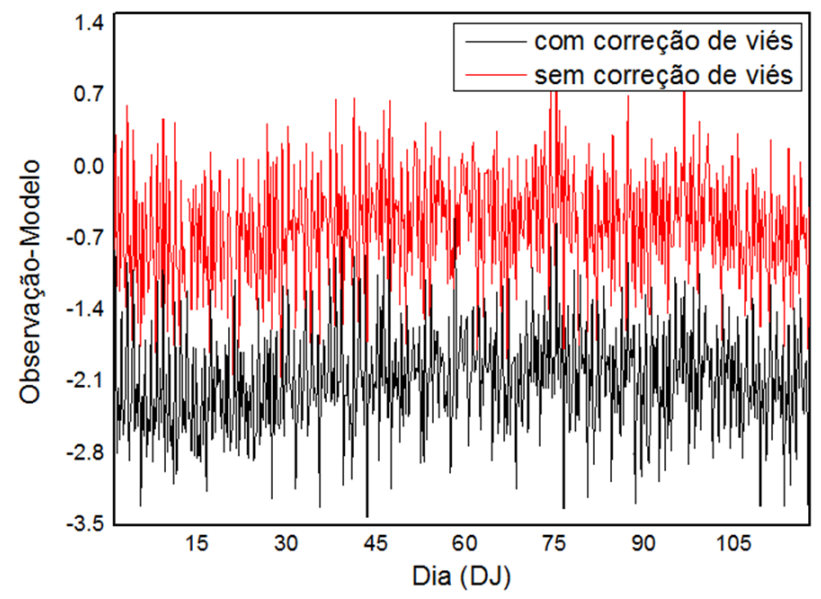

(c)

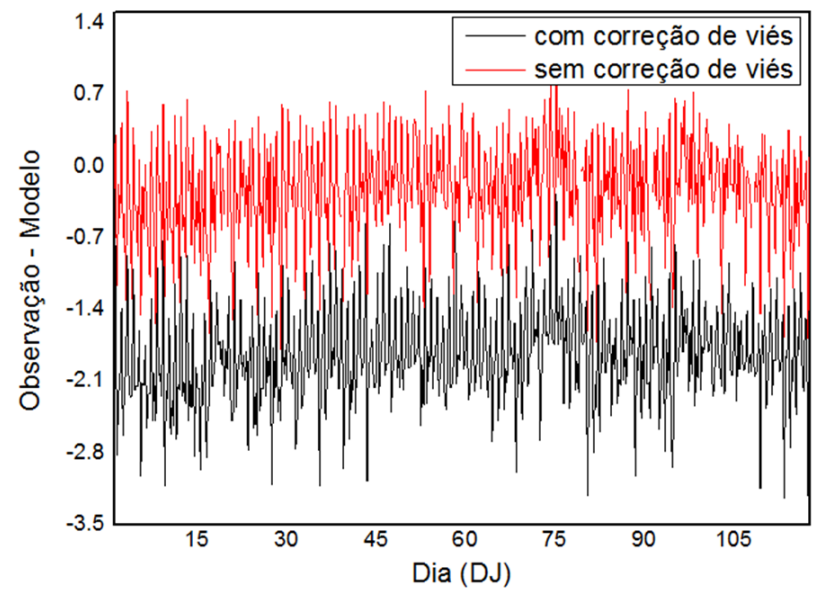

Figura 2: Distribuição temporal das radiâncias para o canal 3 do NOAA-15 sobre a AS: (a) número de observações assimiladas, (b) diferenças entre observada e a estimativa inicial, (c) diferenças entre a observada e a análise. A linha preta indica que foi aplicada a correção de viés do satélite, enquanto que a linha vermelha representa dados sem correção de viés. 
média, os valores de temperatura de brilho observada foram menores que os da simulada e esta diferença é ampliada após a correção de viés do satélite. Este comportamento permaneceu na comparação entre a observação e a análise (Figura 2c). Esse padrão também foi verificado nos canais 1 e 15 . Todavia, para o canal 2 do satélite NOAA-15, a correção de viés não ampliou a diferença.

Para o satélite NOAA-18 (não mostrado aqui), o número de observações assimiladas oscilou em torno de no mínimo 100 no canal 2, 125 no canal 1 e 200 nos canais 3 e 15 . A passagem ocorreu às 0600 e $1800 \mathrm{Z}$ e cobriu toda a região. A diferença entre a observação e a simulação foi maior no canal 3, cerca de $-2 \mathrm{~K}$ ao longo do período. A correção de viés produziu uma redução na diferença entre a observação e a simulação para os todos os canais, exceto para o canal 15 , muito embora essa diferença seja inferior a $-1 \mathrm{~K}$.

Para o satélite NOAA-19 (Figura 3) o horário e a área de cobertura foram semelhantes àqueles verificados com $o$ NOAA-18. O número de observações assimiladas variou em torno de 125 para os canais 1 e 2 e 200 para os canais 3 e 15 . Com relação às temperaturas de brilho observada e simulada, a correção de viés ampliou diferença somente no canal 15 , semelhante ao verificado com o satélite NOAA-18.

Estes aumentos nos valores das diferenças entre as temperaturas de brilho observada e simulada, após a correção de viés, não foram verificados com a introdução dos dados observacionais sobre os oceanos. Assim, as diferenças ficaram restringidas às áreas continentais.

\subsection{Temperaturas de Brilho Simulada}

O gráfico de dispersão da temperatura de brilho simulada contra a temperatura de superfície terrestre forneceu uma ferramenta de visualização quanto à resposta do modelo de transferência radiativa à estimativa inicial fornecida pelo modelo de PNT. Sobre o conjunto de dados os satélites apresentaram concordância, muito embora alguns tenham apresentado uma dispersão maior (Canal 1 e 2 do NOAA-18). A Figura 4 mostra o comportamento para os três satélites no canal 2, o qual se torna linear à medida que os pontos se alinham paralelamente à reta $\mathrm{Y}^{\prime}=\mathrm{X}^{\prime}$.

A estimativa inicial da temperatura de brilho (na ordenada), quando comparada ao produto entre emissividade e a temperatura da superfície terrestre (na abscissa), apresentou um melhor ajuste linear, conforme mostrado na Figura 5 para o satélite NOOA-15, para todos os canais sensíveis à superfície. Nesses casos, esta comparação mostra que o primeiro termo da equação da temperatura de brilho é o que fornece maior contribuição, no mínimo para uma transmitância alta. Por conseguinte, os dois últimos, os quais dizem respeito à emissão
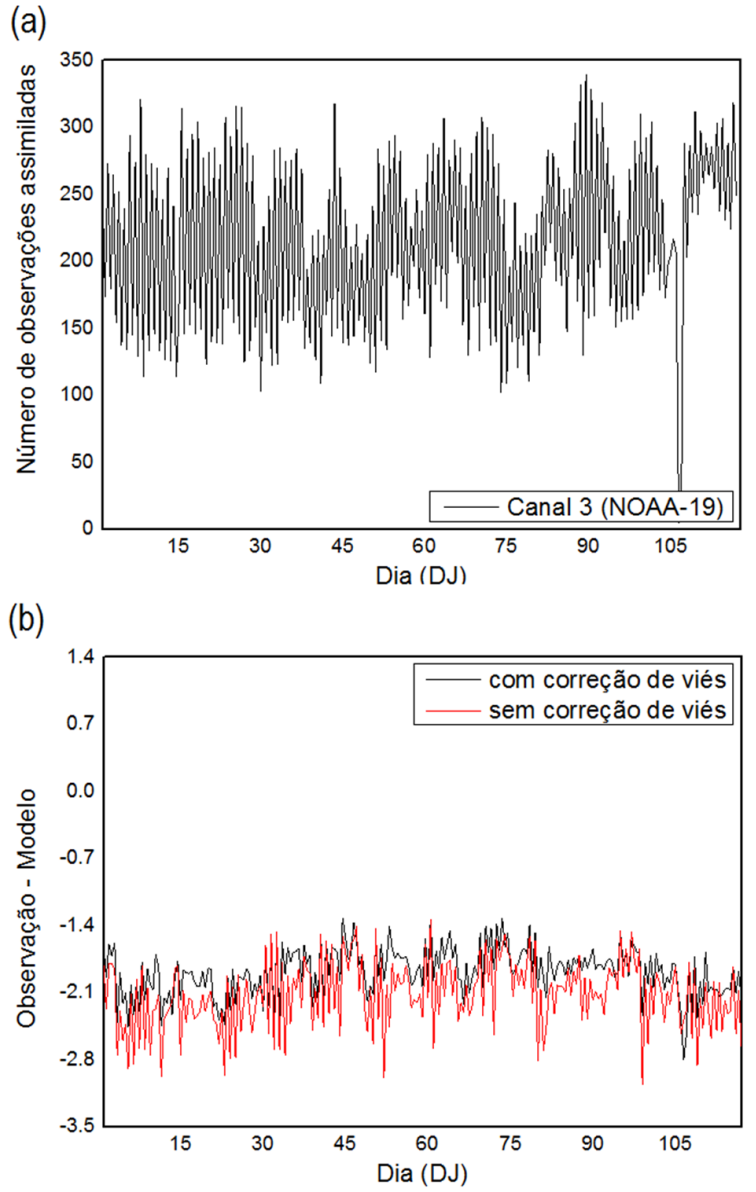

(c)

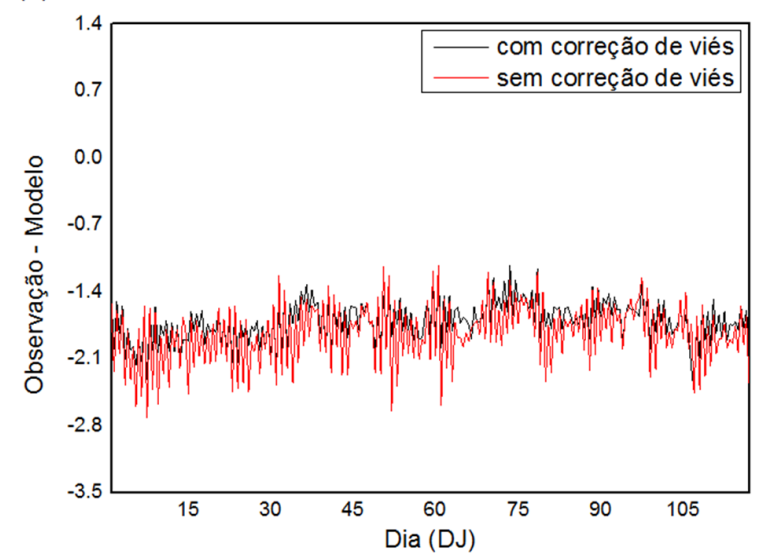

Figura 3: Idem Figura 2, para o NOAA-19.

da radiância através da atmosfera, estão em equilíbrio e sua contribuição é pequena. Esses resultados concordam com os encontrados em Zheng et al. (2012).

Adicionalmente, a transmissividade através da atmosfera também exerce um papel importante (English et al., 2008). Assim, para os canais 3 e 15 dos satélites NOAA-18 e NOAA19 , as diferenças verificadas entre os valores na ordenada 
(a) NOAA-15

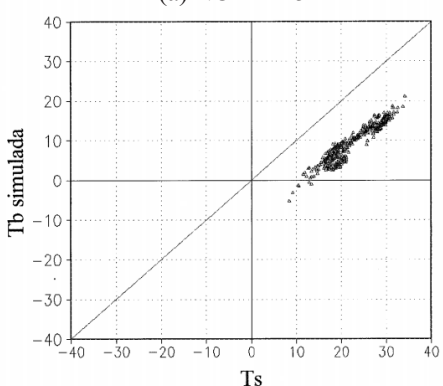

(b) NOAA-18

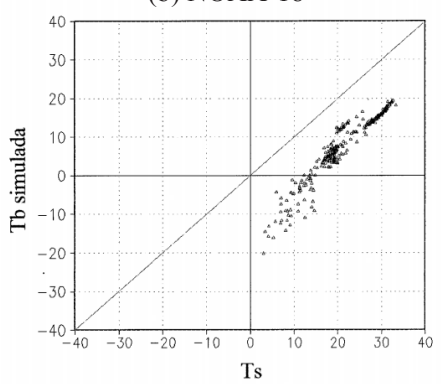

(c) NOAA-19

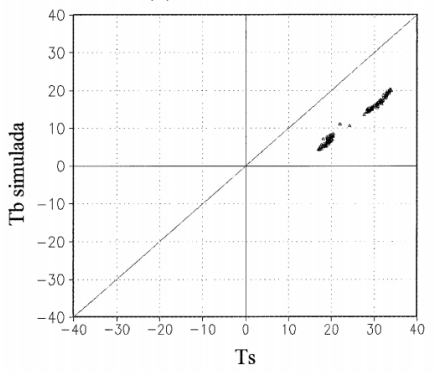

Figura 4: Estimativa inicial da temperatura de brilho no canal 2 (Tb simulada) versus temperatura da superfície terrestre (Ts) para os satélites: (a) NOAA-15; (b) NOAA-18; (c) NOAA-19. A linha que cruza os eixos é a reta $Y^{\prime}=X^{\prime}$.

(a) Canal 1

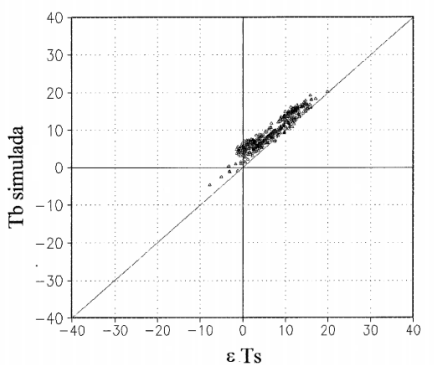

(c) Canal 3

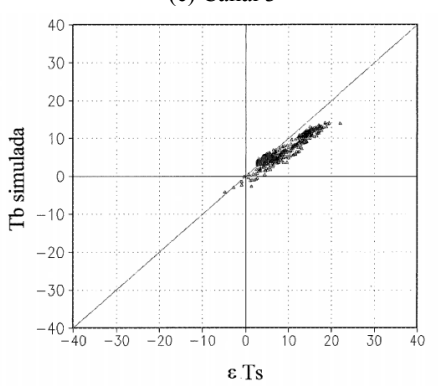

(b) Canal 2

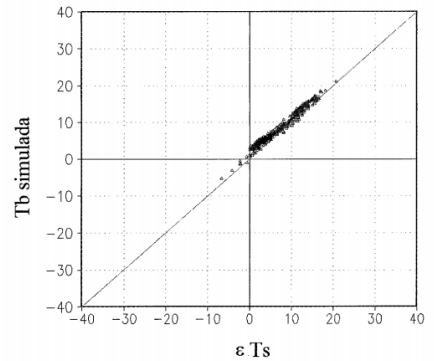

(d) Canal 15

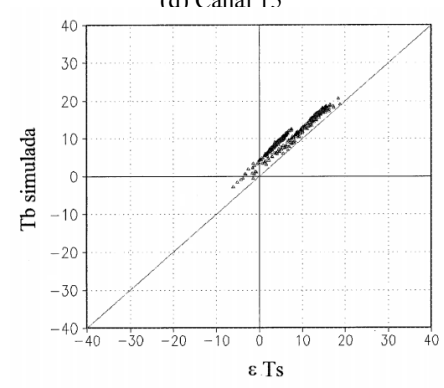

Figura 5: Estimativa inicial da temperatura de brilho para o satélite NOOA-15 (Tb simulada) versus عTs: (a) Canal 1; (b) Canal 2; (c) Canal 3; (d) Canal 15. \&T, o produto entre a emissividade e a temperatura da superfície terrestre. A linha que cruza os eixos é a reta $\mathrm{Y}^{\prime}=\mathrm{X}^{\prime}$.

e na abscissa podem ser justificadas por esse parâmetro. English et al. (2008) classificaram os erros das análises da temperatura de brilho no sensor AMSU-A com base nos valores da transmitância. Quando a transmitância é baixa, os erros significativos na temperatura de brilho provêm grande parte dos erros de temperatura da superfície terrestre; quando é alta, os erros da emissividade da superfície também contribuem.
A Figura 6 apresenta casos onde verificou-se dispersão de dados com relação a curva $\mathrm{Y}^{\prime}=\mathrm{X}^{\prime}$. Em particular, para o satélite NOAA-19, no canal 15, o distanciamento à curva esteve concentrado no conjunto de valores de temperatura de brilho inferior a $10^{\circ} \mathrm{C}$ (Figura 6c). No geral, a relação foi estabelecida em todos os satélites. Todavia, a melhor concordância foi verificada com o satélite NOAA-15, no canal 2, e a pior com 
Tabela 3 Tabela das estatísticas médias durante o período de estudo sobre a AS.

\begin{tabular}{|c|c|c|c|c|c|c|c|c|c|c|c|c|c|}
\hline & \multirow[b]{3}{*}{ Previsão } & \multicolumn{12}{|c|}{ Satélites } \\
\hline & & \multicolumn{8}{|c|}{ NOAA-18 } & \multicolumn{4}{|c|}{ NOAA-19 } \\
\hline & & ges & ges & ges & anl & ges & ges & ges & anl & ges & ges & ges & anl \\
\hline & $\begin{array}{c}\text { Correção } \\
\text { no dado }\end{array}$ & $\mathrm{SCV}$ & $\mathrm{CCV}$ & CQ & CQ & SCV & $\mathrm{CCV}$ & CQ & $\mathrm{CQ}$ & SCV & $\mathrm{CCV}$ & CQ & CQ \\
\hline \multirow{2}{*}{$\begin{array}{c}\text { Canal } \\
1\end{array}$} & Y/X & 1,01 & 1,01 & 1,01 & 1,02 & 1,01 & 1 & 1 & 1,01 & 1,01 & 1 & 1 & 1,01 \\
\hline & REQM & 3,18 & 4,24 & 4,61 & 5,47 & 2,85 & 2,2 & 3,18 & 3,68 & 2,69 & 1,62 & 1,81 & 2,7 \\
\hline \multirow{2}{*}{$\begin{array}{c}\text { Canal } \\
2\end{array}$} & $\mathrm{Y} / \mathrm{X}$ & 1 & 1 & 1 & 1 & 1 & 1 & 0,99 & 1 & 1 & 1 & 0,99 & 1 \\
\hline & REQM & 1,52 & 0,92 & 2,1 & 1,52 & 1,4 & 1,17 & 2,87 & 2,52 & 1,27 & 1,43 & 2,56 & 1,71 \\
\hline \multirow{2}{*}{$\begin{array}{c}\text { Canal } \\
\mathbf{3}\end{array}$} & Y/X & 0,99 & 1 & 1 & 1 & 0,99 & 0,99 & 0,99 & 0,99 & 0,99 & 0,99 & 0,99 & 0,99 \\
\hline & REQM & 2,89 & 1,85 & 1,99 & 2,21 & 3,96 & 4,66 & 4,62 & 4,15 & 4,03 & 3,95 & 4,26 & 3,78 \\
\hline \multirow{2}{*}{$\begin{array}{c}\text { Canal } \\
15\end{array}$} & Y/X & 1,01 & 1,02 & 1,02 & 1,03 & 1,01 & 1,02 & 1,02 & 1,02 & 1,01 & 1,01 & 1,02 & 1,02 \\
\hline & REQM & 3,67 & 6,92 & 6,44 & 7,57 & 3,48 & 4,96 & 5,3 & 6,15 & 3,28 & 4,46 & 4,92 & 6,13 \\
\hline
\end{tabular}

$\mathrm{X}$ :Emissividade * Temperatura da Superfície Terrestre; Y: Temperatura de Brilho Simulada; SCV: Sem correção de viés do satélite e sem controle de qualidade, CCV: Com correção de viés do satélite e sem controle de qualidade; CQ: com controle de qualidade após a correção de viés do satélite; REQM: Raiz do Erro Quadrático Médio entre $X$ e $Y \sqrt{(X-Y)^{2}}$.

(a) NOAA-18, canal 3

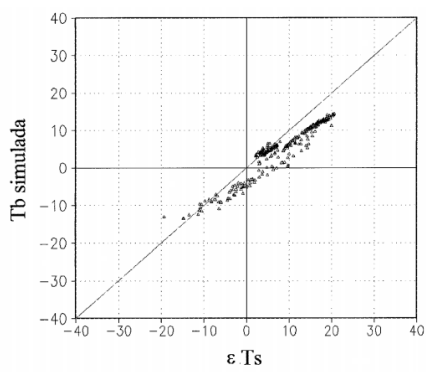

(b) NOAA-19, canal 3

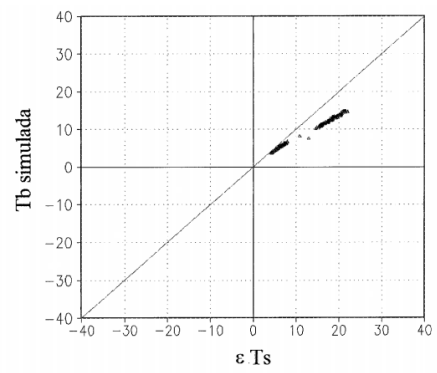

(c) NOAA-19, canal 15

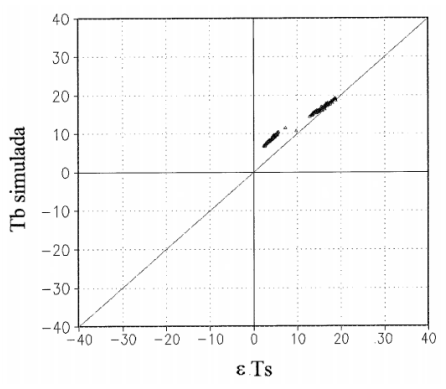

Figura 6: Idem a Figura 5: (a) NOAA-18, canal 1; (b) NOAA-19, canal 2; (c) NOAA-19, canal 15.

o satélite NOAA-15 no canal 15 , conforme as estatísticas apresentadas na Tabela 3, como a relação $\mathrm{Y} / \mathrm{X}$ e a raiz do erro quadrático médio. As informações na Tabela 3 incluem os valores das radiâncias simuladas para a estimativa inicial e a análise e, conforme verificado, os valores diferem nesses dois casos. Isso se deve a perda de dados devido ao processo de controle de qualidade, à seleção dos dados observados a qual diminui a amostragem.

\section{CONCLUSÕES E RECOMENDAÇÕES}

As radiâncias para os satélites NOAA-15, NOAA18 e NOAA-19 para os canais sensíveis à superfície foram investigadas no Sistema de Assimilação de Dados G3DVAR do CPTEC/INPE (não operacional). Os resultados mostraram que as radiâncias observadas foram assimiladas satisfatoriamente em todos os satélites, com um número de aceite maior para os 
dados dos satélites NOAA-18 e NOAA-19, indicando assim, que estes podem ser incluídos no modo operacional do Sistema G3DVAR.

Quanto à temperatura de brilho simulada para os canais sensíveis à superfície terrestre para os três satélites na região da $\mathrm{AS}$, em média, ela é superestimada com relação às observações. Consequentemente, para este mesmo período, em média, a temperatura da superfície terrestre está sendo superestimada, pois como averiguado, estes dois parâmetros possuem um comportamento linear, ou seja, um aumento da temperatura da superfície terrestre ocasiona um aumento da temperatura de brilho simulada e vice-versa. Além disso, o produto da emissividade e temperatura da superfície terrestre praticamente descreve a temperatura de brilho simulada nos canais sensíveis à superfície terrestre do sensor AMSU-A. Isto indica a importância da boa representação da temperatura da superfície terrestre e da emissividade em modelos numéricos que visam assimilar radiâncias nesses canais.

Ressalta-se que durante o controle de qualidade da radiância observada, a estimativa inicial é considerada como "verdade". Como esta medida contém erros inerentes ao próprio modelo de PNT, há necessidade de validação desses dados. Esse aspecto está sendo investigado e é objeto de artigo seguinte, em elaboração. Ademais, sugere-se uma investigação do papel da topografia, como uma forçante externa, na radiância, uma vez que a região da $\mathrm{AS}$ inclui a Cordilheira dos Andes em toda a sua extensão.

\section{AGRADECIMENTOS}

Os autores agradecem ao Grupo de Assimilação de Dados do CPTEC / INPE pelo apoio indispensável. Ao Conselho Nacional de Desenvolvimento Científico e Tecnologia, pelo apoio financeiro.

\section{REFERÊNCIAS BIBLIOGRÁFICAS}

ARAVEQUIA, J. A. A assimilação de radiâncias no filtro de Kalman local por conjunto. In: CONGRESSO BRASILEIRO DE METEOROLOGIA, 15, São Paulo. Anais... 2008.

BONATTI, J. P. Modelo de circulação geral atmosférico do CPTEC. Climanálise Especial (10 anos Edição Especial), 1996.

ENGLISH, Stephen J. The importance of accurate skin temperature in assimilating radiances from satellite sounding instruments. Geoscience and Remote Sensing, IEEE Transactions on, v. 46, n. 2, p. 403-408, 2008.

GONÇALVES ET AL., 2015. Overview of the Global 3DVAR Data Assimilation System at CPTEC/INPE. (artigo em elaboração)
HAN, Y.; VAN DELST P.; LIU, Q.; WENG, F.; DERBER, J. C. User's guide to the JCSDA community radiative transfer model (beta version). Joint Center for a Sattelite Data Assimilation, Camp Springs, Maryland, USA, 2005.

HE, W.; LIU. Z.; CHEN, H. Influence of Surface Temperature and Emissivity on AMSU-A Assimilation over Land. Acta Meteorologica Sinica, v. 5, p. 003, 2011.

HU, M.; SHAO, H.; STARK, D.; NEWMAN, K. User's Guide to Gridpoint Statistical Interpolation (versão 3.2). National Center for Atmospheric Research, National Centers for Environmental Prediction and Earth System Research Laboratory. July 2013.

HERDIES, D. L., ARAVÉQUIA, J. A., FERREIRA, S. H. S., ANDREOLI, R. V., SAPUCCI, L. F., \& MATTOS, J. G. Z.. A Assimilação de Dados no CPTEC/INPE. Boletim da Sociedade Brasileira de Meteorologia, v. 32, p. 57-64, 2008.

KALNAY, E. Atmospheric modeling, data assimilation, and predictability. Cambridge university press, 2003.

KARBOU, F.; BORMANN, N.; THÉPAUT, J. N. Towards the assimilation of satellite microwave observations over land: Feasibility studies using SSMI/S, AMSU-A and AMSU-B.ECMWF, Reading, UK, NWP SAF Tech. Rep, 2007.

KARBOU, F.; GÉRARD, E.; RABIER, F. Microwave land emissivity and skin temperature for AMSU-A and-B assimilation over land. Quarterly Journal of the Royal Meteorological Society, v. 132, n. 620, p. 2333-2355, 2006.

KAZUMORI, M. Assimilation experiments involving surface-sensitive microwave radiances in JMA's global data assimilation system. CAS/JSC WGNE Activities in Atmospheric and Oceanic Modelling, 2012.

KLEIST, Daryl T. et al. Introduction of the GSI into the NCEP global data assimilation system. Weather and Forecasting, v. 24, n. 6, p. 1691-1705, 2009.

KUBOTA, P. Y. Variabilidade da energia armazenada na superfície e o seu impacto na definição do padrão de precipitação na América do Sul.Tese (Doutorado em Meteorologia) - Instituto Nacional de Pesquisas Espaciais (INPE), São José dos Campos, 2012.

LIOU, K. N. An introduction to atmospheric radiation. Academic press, 1980

MEDEIROS, M. D. S. Impacto do uso de radiância na assimilação de dados usando 4D-LETKF na região da América do Sul. Dissertação (Mestrado em Meteorologia) - Instituto Nacional de Pesquisas Espaciais, São José dos Campos, 2010.

SILVEIRA, B.; GONÇALVES, L. G.; BASTARZ, C. F.; AZEVEDO, H. B. O uso de radiâncias na assimilação de dados globais 3 dvar do cptec/inpe. In: CONGRESSO 
BRASILEIRO DE METEOROLOGIA, 17., Gramado. Anais... Rio de Janeiro: SBMET, 2012. 37

SOUZA, S. S.; ET AL. Impact of the scheme of deep convection in the global data assimilation system LETKF of the CPTEC/ INPE. In: THE MEETING OF THE AMERICAS, 2010, Foz do Iguaçu, BR. Proceedings... 2010.

XUE, Y.; SELLERS, P. J.; Kinter, J. L.; Shukla., J. A simplified biosphere model for global climate studies. Journal of Climate, v.4, p. 345-364, 1991.

WENG, Fuzhong; YAN, Banghua; GRODY, Norman C. A microwave land emissivity model. Journal of Geophysical Research: Atmospheres (1984-2012), v. 106, n. D17, p. 20115-20123, 2001.
ZHENG, W.; WEI, H.; MENG, J.; EK, M.; MITCHELL, K.; and DERBER, J. Improvement of land surface skin temperature in NCEP operational NWP models and its impact on satellite data assimilation. In: 23rd Conference on Weather Analysis and Forecasting/19th Conference on Numerical Weather Prediction. 2009

ZHENG, W.; WEI, H.; WANG, Z.; ZENG, X.; MENG, J.; EK. M.; MITCHELL, k.; e DERBER, J. Improvement of daytime land surface skin temperature over arid regions in the NCEP GFS model and its impact on satellite data assimilation. Journal of Geophysical Research: Atmospheres (1984-2012), v. 117, n. D6, 2012. 